\title{
„Hip-Diagnose“: Nicht-Zöliakie-Weizensensitivität
}

\begin{abstract}
Die Abklärung vermeintlicher Nahrungsmittelallergien und Nahrungsmittelunverträglichkeiten gehört zu den großen Herausforderungen in der gastroenterologischen Praxis.
\end{abstract}

Immerhin liege der Anteil der Menschen mit einer Nahrungsmittelintoleranz in der Gesamtbevölkerung bei $20-35 \%$, mit einer Nahrungsmittelallergie bei 2-5\%, erläuterte Professor

\begin{tabular}{|c|c|c|}
\hline & Zöliakie & Weizensensitivität \\
\hline $\begin{array}{l}\text { Zeitraum zwischen } \\
\text { Glutenexposition } \\
\text { und Symptomen }\end{array}$ & Wochen bis Jahre* & Stunden bis Tage \\
\hline Pathogenese & $\begin{array}{l}\text { angeborene und ad- } \\
\text { aptative Immunant- } \\
\text { worten }\end{array}$ & $\begin{array}{l}\text { wahrscheinlich angebore- } \\
\text { nen Immunität gegen z.B. } \\
\text { ATIs; Vermehrung der IEL } \\
\text { möglich (nicht so stark wie } \\
\text { bei klassischer Zöliakie) }\end{array}$ \\
\hline HLA & HLA DQ2/DQ8 in 99\% & nicht bekannt \\
\hline Antikörper & tTG-antikörperpositiv & $\begin{array}{l}\text { Gliadinantikörper } \\
\text { (IgAoder lgG) möglich }\end{array}$ \\
\hline $\begin{array}{l}\text { Dünndarmhistologie } \\
\text { (unter Normalkost) }\end{array}$ & MARSH 2-4 & MARSH 0-1 \\
\hline Komplikationen & $\begin{array}{l}\text { Komorbiditäten, lang- } \\
\text { fristige Komplikatio- } \\
\text { nen (Lymphomrisiko) }\end{array}$ & noch unklar \\
\hline
\end{tabular}

Klaus-Peter Zimmer, Mainz. Bei einem Morbus Crohn oder einer Colitis ulcerosa steige das Risiko auf $45-65 \%$ respektive $5-10 \%$. Derzeitige „Hip-Diagnose“ ist laut Zimmer die NichtZöliakie-Weizensensitivität (NZWS). Besteht anamnestisch der Verdacht auf eine NZWS, müssen vor Diagnosestellung zwingend eine Reihe von Differenzialdiagnosen abgeklärt werden, allen voran die Zöliakie ( $\checkmark T a b .1)$. Einen in der Praxis gangbaren Diagnosealgorithmus stellte Professor Jürgen-Michael Stein, Frankfurt a.M., vor. Danach soll bei Verdacht auf eine NZWS eine Zöliakie mittels Duodenalbiopsie und Serologie abgeklärt werden. Nächster Schritt ist der Ausschluss einer Weizenallergie mittels Prick-Hauttest und Nachweis von spezifischen IgE im Serum. Sind Zöliakie und Weizenallergie nicht, wird eine glutenfreie Diät über mindestens zwei Monate durchgeführt. Bessern sich die Beschwerden, setzt man den Patienten erneut eine Woche glutenhaltiger Nahrung aus. Treten dann erneut Symptome auf, die sich unter eine glutenfreien Diät wieder bessern, kann die Diagnose NZWS gestellt werden. Andernfalls kommen Differenzialdiagnosen in Betracht, insbesondere eine FODMAPUnverträglichkeit. „20\% derjenigen, die über eine Glutenunverträglichkeit klagen, leiden eher unter einer FODMAP-Unverträglichkeit vor", erklärte Stein. Weitere Differenzialdiagnosen zur NZWS sind eine bakterielle Übersiedelung, Fruktose/Laktosemalabsorption sowie mikroskopische Kolitis und Gastroparese. Auch eine chronisch entzündliche Darmerkrankung oder ein Reizdarm kommen in Betracht. Dr. Beate Fessler

Nahrungsmittelallergien und Durchfallerkrankungen: Viszeralmedizin 2017, 14.9.2107 in Dresden

\section{Wenn Medikamente die Speiseröhre schädigen}

\begin{abstract}
Ösophageale Ulzera, die durch oral eingenommene Medikamente induziert werden, sind keine Seltenheit. Bei den Ursachen für diese Läsionen stehen die Medikamente sogar an zweiter Stelle.
\end{abstract}

Etwa $20 \%$ der ösophagealen Läsionen werden durch Medikamente verursacht, so Professor Wolfgang Schepp, München. Sie stehen damit an zweiter Stelle der Ursachen nach der gastroösophagealen Refluxkrankheit mit etwa $65 \%$. Die Inzidenz wird mit 3,0/100.000 Einwohnern pro Jahr angegeben. Etwa zwei Drittel der Patienten sind weiblich, unter anderem, weil sie häufiger schädigende Medikamente wie Bisphosphonate erhalten. An Läsionen im Ösophagus sollte man bei Patienten denken, die über retrosternale Schmerzen, Dysphagie und Odynophagie klagen. Selten geht eine Läsion im Ösophagus auch mit Erbrechen, Hämatemesis, Teerstuhl und Gewichtsverlust einher. Die Symptome treten Stunden bis Tage nach Entstehung der Läsion auf, längstens aber einen Monat nach oraler Einnahme. Lokalisiert ist die Läsion überwiegend im mittleren Ösophagusdrittel. Was aber tun, wenn notwendige Medikamente der Speiseröhre scha- den? Sie sollten abgesetzt oder zumindest auf eine flüssige Formulierung umgestellt werden, erklärte Schepp. Bei ausgeprägter Dysphagie/Odynophagie mit unzureichender oraler Nahrungsaufnahme kann eine parenterale Flüssigkeitszufuhr oder Ernährung notwendig werden. Medikamentös verwies Schepp auf Protonenpumpeninhibitoren, eventuell auch Antazida, $\mathrm{H}_{2}$-Antagonisten oder Lokalanästhetika, jedoch ohne Evidenz. Bei Strikturen kann eine endoskopische Dilatation indiziert sein.

\section{Bei der Tabletteneinnahme ausreichend trinken}

Besser ist allerdings die Prävention solcher Läsionen. Bei Patienten mit vorbestehender Ösophaguserkrankung, wie GERD, Stenose oder Achalasie, bei dilatiertem linken Vorhof oder thorakalem Aortenaneurysma sollten ösophagotoxische Medikamente restriktiv verordnet werden. Wichtig: Die Patienten sollten generell ermahnt werden, bei der Tabletteneinnahme ausreichend zu trinken. Schepp empfahl mindestens $50-240 \mathrm{ml}$ Flüssigkeit.

Dr. Beate Fessler

Medikamenten-induzierte Schäden der Verdauungsorgane: Viszeralmedizin 2017, 15.9.2107 in Dresden 